\title{
Prediction of the Dst index from solar wind parameters by a neural network method
}

\author{
Shigeaki Watanabe, Eiichi Sagawa, Kazuhiro Ohtaka, and Hironori Shimazu \\ Communications Research Laboratory, Koganeishi, Tokyo 184-8795, Japan
}

(Received June 1, 2001; Revised October 17, 2002; Accepted October 17, 2002)

\begin{abstract}
Using the Elman-type neural network technique, operational models are constructed that predict the Dst index two hours in advance. The input data consist of real-time solar wind velocity, density, and magnetic field data obtained by the Advanced Composition Explorer (ACE) spacecraft since May 1998 (http://www2.crl.go.jp/uk/uk223/service/nnw/index.html). During the period from February to October 1998, eleven storms occurred with minimum Dst values below $-80 \mathrm{nT}$. For ten of these storms the differences between the predicted minimum Dst and the minimum Dst calculated from ground-based magnetometer data were less than $23 \%$. For the remaining one storm (beginning on 19 October 1998) the difference was $48 \%$. The discrepancy is likely to stem from a imperfect correlation between the solar wind parameters near ACE and those near the earth. While the IMF Bz remains to be the most important parameter, other parameters do have their effects. For instance, Dst appears to be enhanced when the azimuthal direction of IMF is toward the sun. A trapezoid-shaped increase in the solar wind density enhances the main phase Dst by almost $10 \%$ compared with the case of no density increase. Velocity effects appear to be stronger than the density effects. Our operational models have, in principle, no limitations in applicability with respect to storm intensity.
\end{abstract}

\section{Introduction}

Accurate quantitative short-range prediction of space weather has recently become increasingly important because of expanded level of human activity at high latitudes and in space. Several empirical methods of time-series analysis have been used as prediction techniques. Time series analyses are useful because they do not require full understanding of the physical processes involved in the interaction between the solar wind and the magnetosphere. If this relationship were linear, the response of the magnetosphere would not be hard to calculate. If the linearity holds in this relationship, the response can be obtained by convolution of the input data with an empirical impulse-response function. Iyemori et al. (1979) described a useful average linear impulse-response function that shows roughly 40-minute time lags in disturbance indices. Blanchard and McPherron (1992) described an interesting model based on bimodal representation of the response function relating the solar wind electric field to the AL index. In order to take into account of nonlinear response of the magnetosphere to the solar wind, many researchers have tried to obtain accurate geomagnetic disturbance responses - for the Dst, Kp, AE, and AL indices, for example - by using time-series analyses.

The disturbance indices Dst and Dst* have been predicted by the method of Burton et al. (1975), using time varying filter coefficients to incorporate nonlinear responses (e.g., Klimas et al., 1997, 1998), or by using other simulation models in which intense ring current particles are injected from

Copy right (C) The Society of Geomagnetism and Earth, Planetary and Space Sciences (SGEPSS); The Seismological Society of Japan; The Volcanological Society of Japan; The Geodetic Society of Japan; The Japanese Society for Planetary Sciences. the plasma sheet (Ebihara and Ejiri, 1998, 2000). The neural network (NN) method, an artificial intelligence (AI) approach, has also been used to investigate the response of the Dst index to the solar wind. Lundstedt and Wintoft (1994), for example, have shown that a feed-forward NN method with a single hidden layer can predict Dst during geomagnetic storms. Wu and Lundstedt (1997a, b) developed a partially recurrent NN method that during periods of geomagnetic activity, mainly magnetic storms, results in cross correlations of about 0.9 between the predicted values of Dst and the Dst values deduced from actual geomagnetic observation data. For the input parameters, these authors used the solar wind density $\mathrm{N}$ and velocity $\mathrm{V}$, the magnitude of the interplanetary magnetic field (IMF), and the By and Bz components of IMF. Kugblenu et al. (1999) used a multi-layer feed-forward error back-propagation algorithm in their study and obtained good results considering that the training time series consisted of only 20 storms.

In August 1997, NASA launched the Advanced Composition Explorer (ACE) spacecraft into a halo orbit around the first Lagrangian point, 1.50 million $\mathrm{km}(0.01$ astronomical units) from the earth in the direction of the sun. The National Oceanic and Atmospheric Administration makes continuous real-time solar wind data from ACE (Zwickl et al., 1998) available. We have been predicting Dst, using ACE data obtained by the solar wind electron proton alpha monitor (SWEPAM) (McComas et al., 1998) and the magnetic field (MAG) instrument (Smith et al., 1998).

An operational model to be used for prediction has to be constructed for all times even when there are gaps in data, and must produce good predictions at all times. We have 


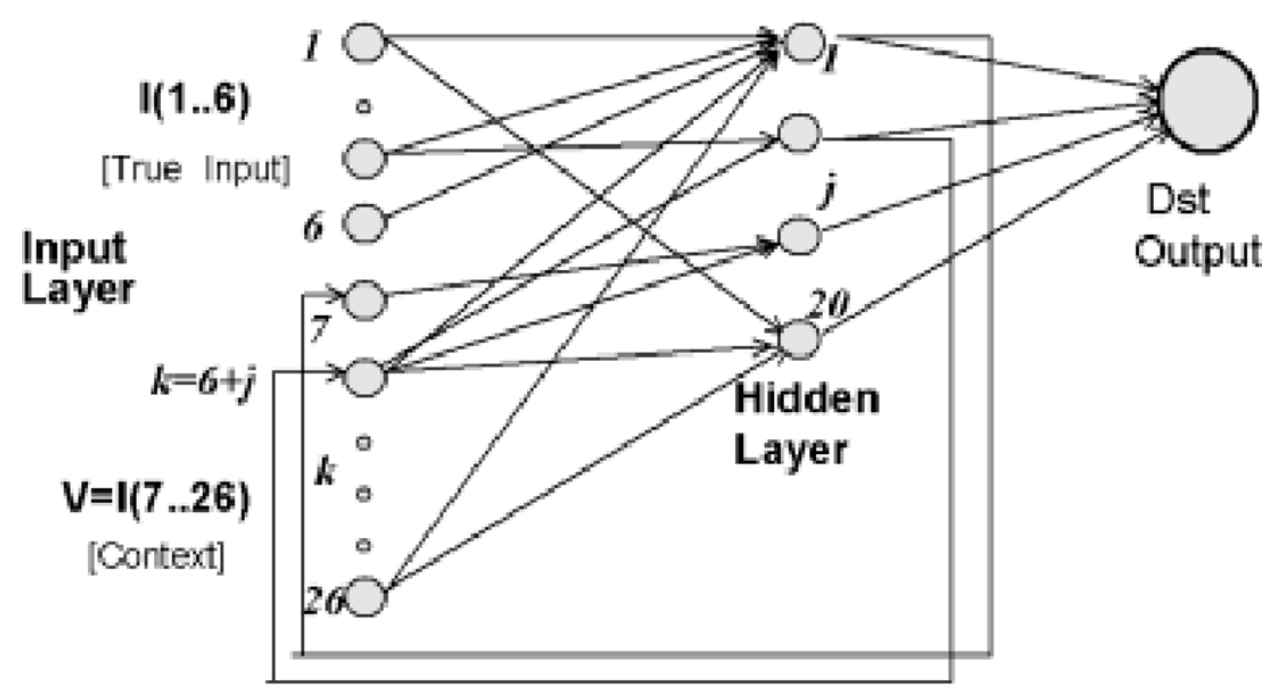

Fig. 1. Architecture of the Elman neural network. (Modified from figure 1 in Wu and Lundstedt, 1997a, b).

therefore constructed many trial models for prediction, and after careful examination and comparison, we have chosen an Elman-type NN model (Elman, 1990; Wu and Lundstedt, 1997a).

In Section 2, we show the equations for the Elman-type $\mathrm{NN}$ model used to predict Dst. We describe in Section 3 the ways in which different versions of this model depend on the conditions of the training time series and explain why we chose the version evaluated in this paper. In Section 4, we discuss the predictions based on this version for the period from February through October 1998. In Section 5, we check the effect of velocity and density, and explain how this version's predicted Dst depends on the direction of the azimuthal angle of the IMF component in the $x-y$ (GSM) plane. In Section 6, we compare the NN model with the Burton type method. In Section 7, we give a summary of our results.

\section{Elman Recurrent NN}

The Elman NN is a two-layer back-propagation network with feedback connections from the hidden layer to its input layer (Elman, 1990; Wu and Lundstedt, 1997a, b). The network architecture is shown in Fig. 1, which is the same as Fig. 1 in Wu and Lundstedt (1997a, b), but modified to suit the present context. The input layer is divided into two parts: the true input units and the context units. The context units hold a copy of the activations of the hidden units from the previous time step. Feedback (or recurrence) usually appears as an integral property in an $\mathrm{NN}$, suggesting that the system has strong time continuation. When $R$ and $S$ are the number of true inputs and the number of hidden units, respectively, and when there is one output unit (Dst), the output of a hidden unit at time $t$ in an input time series is given by the following equations:

$$
\begin{aligned}
& V_{j}^{t}=\tanh \left[\sum_{k=1}^{R} w_{k j} I_{k}^{t}+\sum_{k=R+1}^{R+S} w_{k j} V_{k-R}^{t-1}\right] \\
& D s t=\sum_{j=1}^{S} W_{j} W_{j}^{t} .
\end{aligned}
$$

Where $I_{k}(t)$ is the input of true input unit $k$ at time $t, V_{j}(t-1)$ is the output of hidden unit $j$ in response to the $(t-1)$ th step of the input time series, and $w_{k j}$ is the weight (the connection strength) between input unit $k$ and hidden unit $j . W_{j}$ is the weight (the connection strength) between the hidden unit $j$ and output unit Dst (Eq. (1) is the same as equation (1) in Wu and Lundstedt, 1997a, b).

Constructing an NN model to be used for prediction is equivalent to deciding on a suitable set of $w_{k j}$ and $W_{j}$ values through training involving over 100,000 iterations. Our initial model (model A) used a training time series that included three parameters: the solar wind velocity $V$, solar wind density $N$, and the southward IMF Bz component given in solar magnetospheric coordinates (GSM). At the end of training with a time series obtained from the OMNI data of the National Space Science Data Center (NSSDC/NASA), the cross correlation coefficient between the predicted and observed quantities was 0.89 . Using real-time solar wind (RTSW) data from the ACE spacecraft, prediction by Model A was made open to the public via Web from April to November 1998 (http://www.crl.go.jp/ uk/uk223/service/nnw/index.html). This prediction model performed reasonably well as seen in the cross correlation of 0.89 indicated above. With an intention of improving the prediction we constructed two models (model B, model C) as the next steps, using six parameters in the training time series. Our next two models (models B and C) used six parameters in the training time series.

\section{Dependence of Prediction on the Training Time Series}

The characteristics of the training time-series data for models A, B, and C are listed in Table 1. Models A and B were trained on 5077 hours of data, mainly covering 50 storm periods. Because the operational model has to perform well during periods of moderate activity as well as during periods of strong activity, model $\mathrm{C}$ was trained on 9058 hours of data with 50 active periods including both stormy and moderately active periods. For almost half (44\%) of the time, the magnitude of Dst was less than $50 \mathrm{nT}$ (i.e., Dst was 
Table 1. Characteristics of the training time-series data for three models.

\begin{tabular}{ccccc}
\hline Model & Conditions & Duration & Parameters & Working period \\
\hline A & mainly storm & $5077 \mathrm{~h}$ & Velocity, density, IMF Bs & Version 1 model Apr. 1998-Nov. 1998 \\
B & mainly storm & $5077 \mathrm{~h}$ & Velocity, density, and IMF Bx, By, Bz, Bt & \\
C & storm and quiet & $9058 \mathrm{~h}$ & Velocity, density, and IMF Bx, By, Bz, Bt & Version 2 model Dec. 1998- \\
\hline
\end{tabular}

Bs: southward IMF component $\mathrm{Bz}$ (the northward component was set to 0).

Bt: magnitude (total) of IMF.

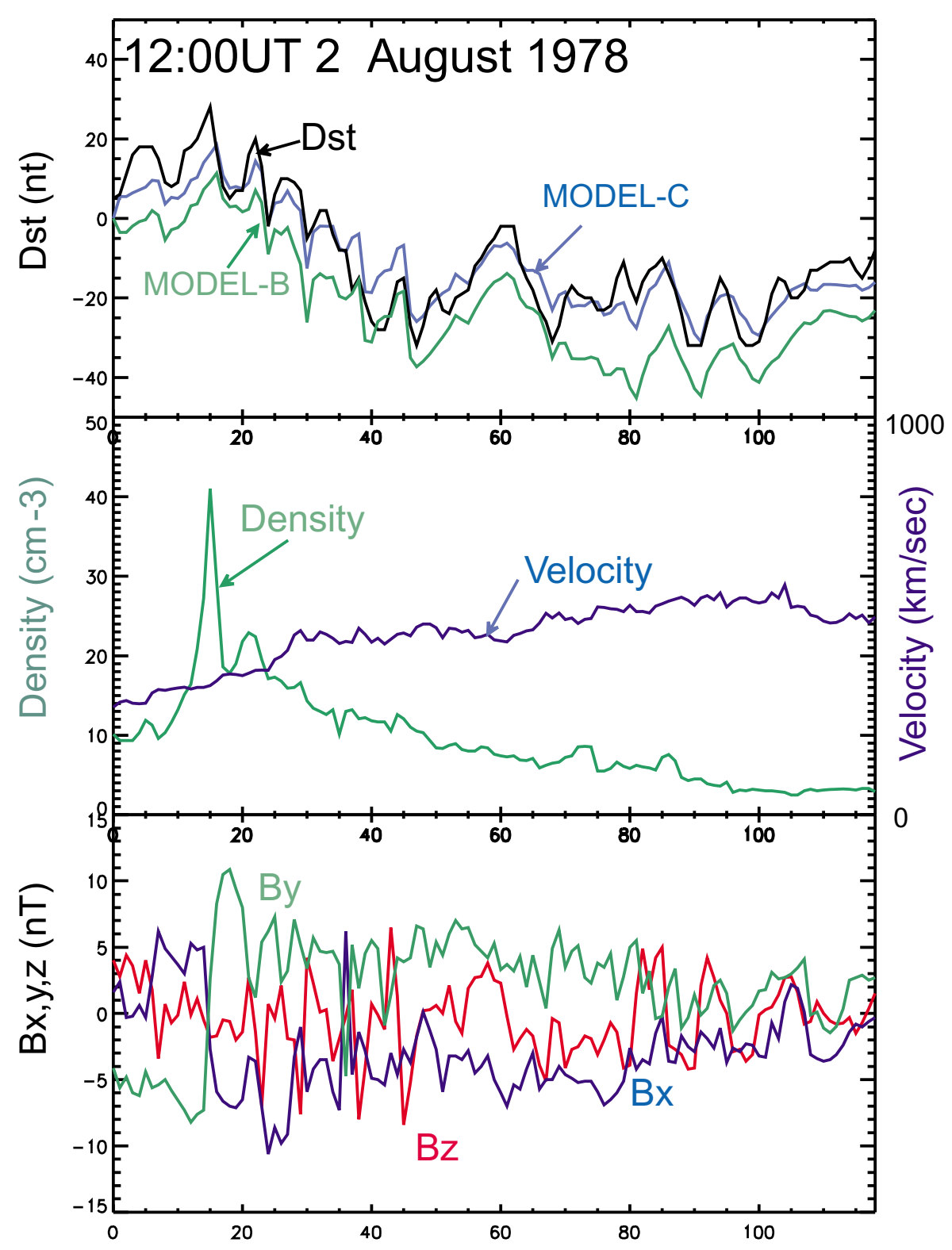

Fig. 2. Dst calculated from ground-based data beginning at 12 UT on 02 August 1978 and the corresponding Dst predicted by models B and C.

greater than $-50 \mathrm{nT})$. We examined models with 10, 20, 30, and 40 hidden units, and the 20 -unit version of model $\mathrm{C}$ gave the best result, the cross correlation coefficient being greater than 0.9 at the end of training. Models B and C were evaluated for periods in 1978 and from February through October of 1998 .

When applied to the RTSW data in the main phase of all storms, the error of the minimum value of Dst predicted by model A two hours in advance was within $23 \%$ of the Dst calculated from the data obtained at ground stations. An Elman-type NN model (models A, B, and C) needs data for a continuous span of time. If calculation starts immediately after the minimum Dst of a storm (i.e., during the recovery phase), the predicted Dst would be at or near moderate levels (Dst $>-50 \mathrm{nT}$ ) because by this time the interplanetary $\mathrm{Bz}$ would have turned northward. Calculations for the predic- 
(nT)

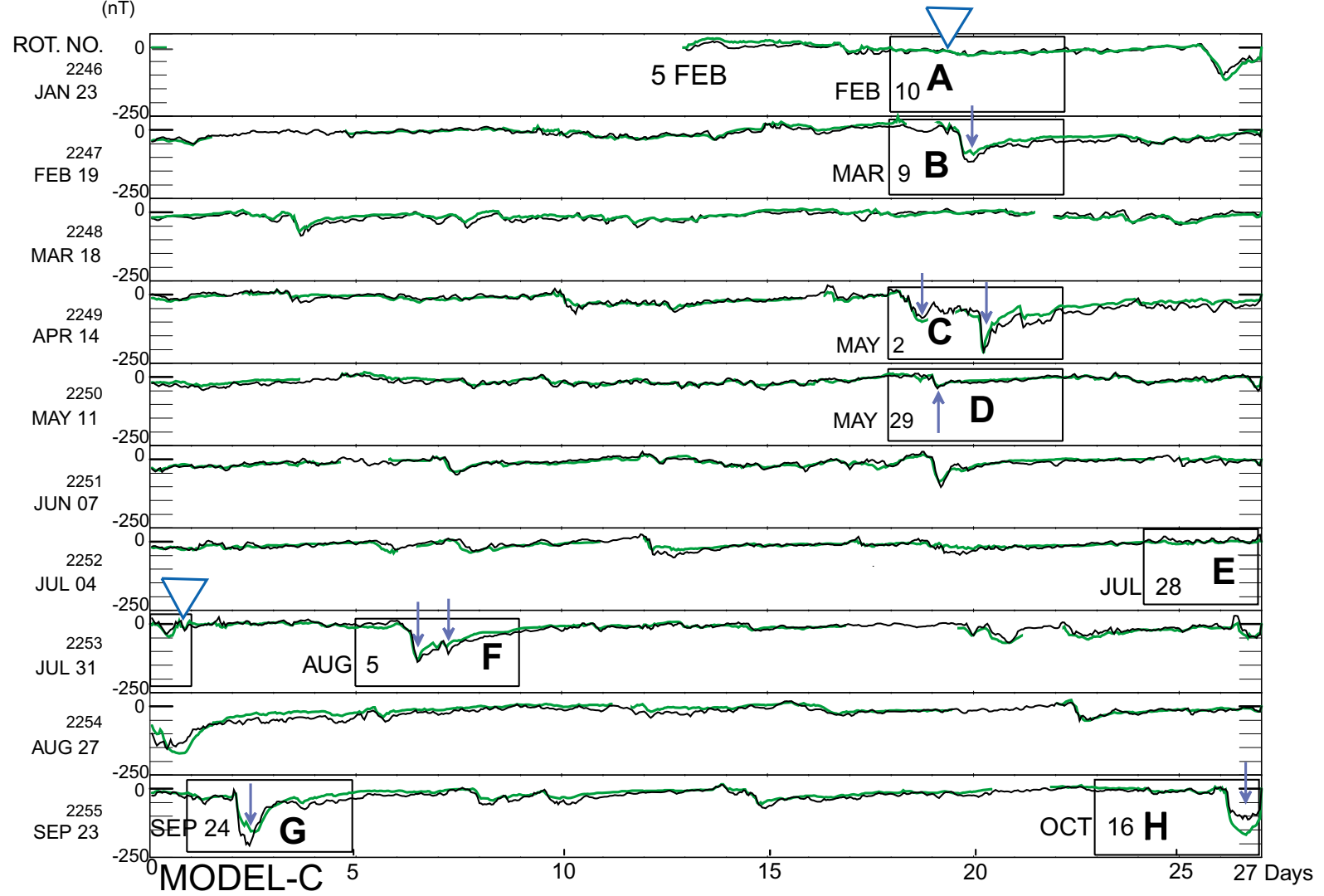

Fig. 3. For each solar rotation (Bartels number) from February to October 1998, Dst calculated from ground-based data (black curve) and Dst predicted by model C (green curve). Periods labeled with capital letters are shown in more detail in Fig. 4.

tion of Dst must therefore start in the quiet period before a magnetic storm.

The performance of the three NN models depended on the characteristics of the data used for the training. An example of a quiet period is shown in Fig. 2; it covers 120 hours starting from 12 UT on 2 August 1978. The black line in the top panel shows the Dst calculated from data obtained at ground stations. The velocity (indicated by the blue curve in the middle panel over the range from 0 to $1000 \mathrm{~km} \mathrm{~s}^{-1}$ ) increases from $270 \mathrm{~km} \mathrm{~s}^{-1}$ at 12 UT on 2 August to 578 $\mathrm{km} \mathrm{s}^{-1}$ at $20 \mathrm{UT}$ on 6 August. The density (plotted in green in the middle panel over the range from 0 to $50 \mathrm{~cm}^{-3}$ ) reaches a peak of about $41 \mathrm{~cm}^{-3}$ at 03 UT on 3 August. The full scale in the bottom panel is $\pm 15 \mathrm{nT}$, and the IMF $\mathrm{Bx}$, $\mathrm{By}$, and $\mathrm{Bz}$ components are shown, respectively, by blue, green, and red lines. This panel shows a situation in which Bz was weakly negative; it covered a quiet/quasi-quiet period (Dst $>-30 \mathrm{nT}$ ).

As shown in the top panel, the curve produced by model $\mathrm{C}$ fits the Dst curve calculated from data recorded by ground stations better than does the curve produced by model B. This indicates that the use of training data, which include moderately active as well as fully active periods, produces a better model. When the final processed solar wind data gathered after February 1998 were used, model C produced more accurate predictions during both active and quiet periods than did models A and B. Since December 1998 we have therefore been using model $\mathrm{C}$ to predict Dst indices from RTSW data.

\section{Prediction Accuracy of Model C}

As has been mentioned, all three models were trained using data gathered during a period of maximum solar activity (1978-1982), but we evaluated model C's ability to predict Dst during a period in which solar activity is increasing (since February 1998). As is shown in Fig. 3, we used data continuously gathered by the ACE spacecraft from February to October 1998. Model $\mathrm{C}$ takes into account both the time it takes for the solar wind to travel 1.5 million $\mathrm{km}$ from the first Lagrangian point to the earth magnetosphere and the response time of the magnetosphere. In Fig. 3, Dst and its predicted values are plotted for each solar rotation, the solar rotation number and the starting date being indicated to the left of each line; the number of days from the start is shown at the bottom. Plotting in this manner, recurrent activity can readily be found. The Final Dst (determined by World Data Center for Geomagnetism, Kyoto, based on data gathered from ground stations) is shown by the black curves in Fig. 3. The Dst predictions by model $\mathrm{C}$, shifted to the right by two hours to compensate for the time lag, are shown by the green curves. We see that the pattern of the predicted Dst follows very closely the actual Dst curve.

During the period covered by Fig. 3, there were eleven storms with a minimum Dst of less than $-80 \mathrm{nT}$. The differences between the minimum predicted Dst and the Dst calculated from the ground data are less than about $23 \%$ for ten of these eleven storms. The remaining one event, starting on 16 October, shows a $48 \%$ difference, which probably is due to a non-perfect correlation of the physical conditions 


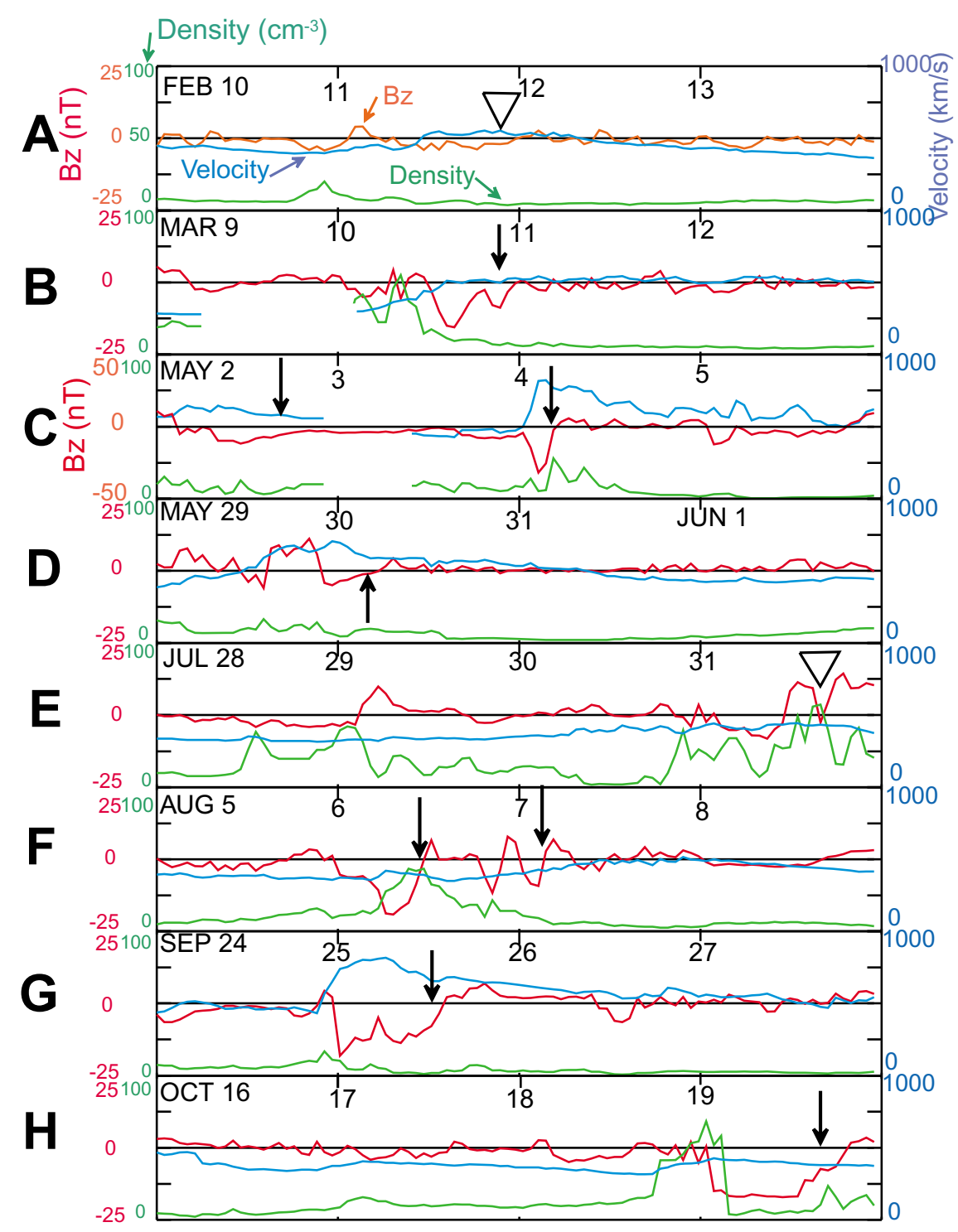

Fig. 4. Solar wind velocity (Blue curve) and density (green curve) and the $Z$ component of the interplanetary magnetic field (red curve) during periods labeled with capital letters, A to $\mathrm{H}$ in Fig. 3. The start date for a four-day time span is indicated in each panel. The arrows show the minimum Dst in the boxed periods of Fig. 3. The downward-pointing triangles indicate noteworthy features.

between the location of ACE (the L1 point) and the vicinity of the Earth's magnetosphere. Apart from this exception, the predicted Dst was in approximate agreement with the actual Dst during various phases of solar activity.

Figure 4 shows more detailed views of the behavior of the solar wind parameters in the eight boxed periods of Fig. 3, each of which spans four days. In each panel of Fig. 4, $V$ (blue curve), $N$ (green curve), and IMF Bz in the GSM coordinates (red curve) are plotted over ranges of, respectively, 0 to $1000 \mathrm{~km} \mathrm{~s}^{-1}, 0$ to $100 \mathrm{~cm}^{-3}$, and -25 to $+25 \mathrm{nT}$, except in panel $\mathrm{C}$ where $\mathrm{Bz}$ is plotted over a range of -50 to $+50 \mathrm{nT}$ because of the greater magnitudes of $\mathrm{Bz}$ during this period. The 0 level for $\mathrm{Bz}$ is indicated by black line in each panel. Gaps in solar wind density and velocity curves indicate no data in panels B and $\mathrm{C}$. The blue and black arrows indicate the Dst minima for the boxed periods of Fig. 3 after adjusting for the time lag of the prediction.

Each Dst minimum occurred towards the end of a negative $\mathrm{Bz}$ valley rather than exactly at the minimum of the $\mathrm{Bz}$. This suggests that $\mathrm{Bz}$ has a decisive effect on negative Dst values.

(10-13 February) Figure 4(A): The density is $\sim 10 \mathrm{~cm}^{-3}$, with a peak at $20 \mathrm{~cm}^{-3}$ at $23 \mathrm{UT}$ on 10 February. A fast solar wind, caused by a coronal hole, reached a velocity of $552 \mathrm{~km} \mathrm{~s}^{-1}$ at 22 UT on 11 February (as indicated by the downward-pointing triangle). Despite the relatively high velocity of the solar wind during this period, Fig. 3 shows that Dst activity was low (quiet: $>-29 \mathrm{nT}$ ) because of the small magnitude of $\mathrm{Bz}$ (about $-3 \mathrm{nT}$ ). As can be seen in Fig. 3, the difference between the predicted and calculated Dst values is very small for this period.

(9-12 March) Figure 4(B): After 03 UT on March 10 (there is a long data gap of solar wind plasma before 03 UT on $10 \mathrm{March}$ ), the velocity increase from $300 \mathrm{~km} \mathrm{~s}^{-1}$ to over $500 \mathrm{~km} \mathrm{~s}^{-1}$ after 15 UT because of the same coronal hole responsible for the solar wind observed one solar rotation earlier (Fig. 4(A)). Two pressure pulses occur at 04 and 09 UT on 10 March corresponding to the solar wind density peaks of greater than 42 and $55 \mathrm{~cm}^{-3}$. Large changes in dynamic 
pressure are more often caused by changes in density than changes in velocity. Bz turned to the south at 13 UT and is $-15 \mathrm{nT}$ at $15 \mathrm{UT}$ on $10 \mathrm{March}$; it stayed below $-11 \mathrm{nT}$ for 3 hours. Around the peak of the disturbance (at the start of the recovery phase indicated by the arrow in Figs. 3 and 4(B)), Dst is $-116 \mathrm{nT}$ at $20 \mathrm{UT}$ March 10 . The prediction difference was about $22 \%$ in this event.

(2-5 May) Figure 4(C): This period included one of the greatest disturbances in 1998. There are two peaks in the solar wind velocity which reaches $646 \mathrm{~km} \mathrm{~s}^{-1}$ at $09 \mathrm{UT}$ on 2 May and $824 \mathrm{~km} \mathrm{~s}^{-1}$ at $04 \mathrm{UT}$ on 4 May. The two principal valleys of $\mathrm{Bz}$, whose minima are $-12 \mathrm{nT}$ at $12 \mathrm{UT}$ on 2 May and $-32 \mathrm{nT}$ at 03 UT on 4 May, correspond to the two valleys of Dst which seem to be caused by a coronal hole and a magnetic cloud. The minimum Dst and the difference in the prediction for these events are, respectively, $-85 \mathrm{nT}$ and $22 \%$ at 17 UT on 2 May and $-205 \mathrm{nT}$ and $21 \%$ at $05 \mathrm{UT}$ on 4 May, as each indicated by a downward-pointing arrow in Fig. 3(C).

(29 May to 1 June) Figure 4(D): The velocity of the solar wind increases to $705 \mathrm{~km} \mathrm{~s}^{-1}$ at 00 UT on 30 May because of a solar flare. A plateau lasting over seven hours can be seen in the earlier Bz readings. The shallow valley that followed had a minimum at $-5.0 \mathrm{nT}$. Thereafter the general trend for $\mathrm{Bz}$ is toward zero and weak positive values. Both the predicted Dst and the Dst calculated from measurements at ground stations are small despite the very high solar wind velocity. As can be seen in Fig. 3, the difference between these values was very small. That is, model $\mathrm{C}$ performed well.

(28-31 July) Figure 4(E): This period has one of the largest solar wind densities in 1998. The peak density is 57.3 $\mathrm{cm}^{-3}$ at $16 \mathrm{UT}$ on 31 July. Corresponding to the positive levels of Bz, $11.4 \mathrm{nT}$ and $14.4 \mathrm{nT}$ at $13 \mathrm{UT}$ and $19 \mathrm{UT}$ on 31 July, the predicted Dst values and the Dst values calculated from ground measurements were very small, as was the difference between them.

(5-8 August) Figure 4(F): The density has a peak of 43 $\mathrm{cm}^{-3}$ at $10 \mathrm{UT}$ on 6 August. Despite the almost constant solar wind velocity $\left(360-500 \mathrm{~km} \mathrm{~s}^{-1}\right)$, three deep valleys of $\mathrm{Bz}(-19.2 \mathrm{nT}$ at $08 \mathrm{UT}$ and $-11.7 \mathrm{nT}$ at $21 \mathrm{UT}$ on 6 August, and $-9.2 \mathrm{nT}$ at $03 \mathrm{UT}$ on 7 August) caused one large major storm and a superimposed minor storm with Dst of $-138 \mathrm{nT}$ at $11 \mathrm{UT}$ on 6 and $-108 \mathrm{nT}$ at $05 \mathrm{UT}$ on 7 August, as indicated by the two blue arrows in Fig. 3 which correspond to two black arrows in Fig. 4(F). The difference at 11 UT between the predicted Dst and the Dst calculated from ground measurements was only $6 \%$.

(24-27 September) Figure 4(G): The enormously high velocity (peak: $819 \mathrm{~km} \mathrm{~s}^{-1}$ ) and the long period of highly negative $\mathrm{Bz}$ (peak: $-18 \mathrm{nT}$ ) indicate a large violent storm. The Dst predicted for 09 UT on 25 September $(-169 \mathrm{nT})$ is within $18 \%$ of the actual Dst for that time $(-207 \mathrm{nT})$.

(16-19 October) Figure 4(H): Velocity is almost uniform throughout this period. The density had a large peak, 68 $\mathrm{cm}^{-3}$ at 01 UT on 19 October, before Bz turned from a positive value to an extremely large negative value $(<-11$ $\mathrm{nT}$ ). This is one of the largest and longest lasting negative values in 1998. The Bz measured by ACE remained below $-11 \mathrm{nT}$ from $02 \mathrm{UT}$ to $15 \mathrm{UT}$ on 19 October and then slowly recovered to a positive value at $20 \mathrm{UT}$. On the other hand, the WIND spacecraft (located near ACE at $X s e=95 \mathrm{Re}, Y s e=$ $32 \mathrm{Re}$, and $Z s e=6 \mathrm{Re}$ ) measured $\mathrm{Bz}$ values below -15 nT from 02 to 15 UT. Correspondingly, the Dst prediction using the ACE (and WIND) data indicates (reasonably) a large storm of $-166 \mathrm{nT}$, but the Dst determined from ground stations is smaller, only $-112 \mathrm{nT}$. The predicted Dst differed by $\sim 48 \%$ from the Dst calculated from ground-based data. Thus the correlation between the environment of the ACE spacecraft and that of the earth may not have been so good.

\section{Effects of the Solar Wind Parameters}

Whether or not NN methods are effective for events that are very different from those in the training patterns is a difficult question. However, a good NN model trained on a statistically broad range of natural events should reflect the properties of actual events. Conversely, this supposition confirms the model's prediction capability. A model that effectively predicts a majority of storms should be a good simple simulator for various storm patterns. This capability is very important for reflecting the effects of solar wind parameters, which may be buried under the stronger effects of other parameters such as Bz. We want to be able to isolate the effect of a parameter from the effects of other parameters. However, it is practically impossible to find and compare two actual storms that have a very similar pattern in terms of all but one of their components. Our predictions were within about $20 \%$ of the values calculated from actual data. This discrepancy may be due to an imperfect correlation between the solar wind parameters at the earth and near the L1 point in 1998-1999. We may add that the cross correlation coefficient between the actual Dst and the Dst predicted from the solar wind parameters near the earth was over 0.91 for the time interval from 1978 to 1982 including quiet, non-storm periods.

We now examine the effects of solar wind parameters on the effectiveness of an NN model. Because the velocity and the density are major parameters of the solar wind, representative values of these parameters are used in the analysis. In the first trial we set the velocity $(V)$, density $(N)$, and IMF $\mathrm{Bx}$ and By at constant values of $400.0 \mathrm{~km} \mathrm{~s}^{-1}, 3 \mathrm{~cm}^{-3}$, and 10 and $0 \mathrm{nT}$, respectively. As illustrated by the dotted curves in Figs. 5(B) and 5(C) and the solid curve in Fig. 5(D), Bz changes its sign at point $\mathrm{S}$ and drops to $-12 \mathrm{nT}$ over a 2 hour interval, stays at $-12 \mathrm{nT}$ for 15 hours, and then recovers to $+3 \mathrm{nT}$ over a 2 hour period with no change in $N$ or $V$. The computed result for Dst is shown as a dotted curve in Fig. 5(A). This type of storm is often encountered. Because the dynamic pressure does not increase, the calculated Dst curve does not show a pressure pulse (initial phase) produced by an enhancement of the Chapman-Ferraro current. In this case, the minimum Dst of about $-130 \mathrm{nT}$ was near the end of the negative $\mathrm{Bz}$. In other words, Dst continued to decrease until close to the time when the direction of $\mathrm{Bz}$ turned northward from southward as seen in the actual cases shown by the arrows in Fig. 3 and in panels B, C, D, F, G, H of Fig. 4.

Next we perform a simple simulation that takes into account the variation in $N$ and $V$. The simulation should resemble typical cases which have changes in dynamic pres- 


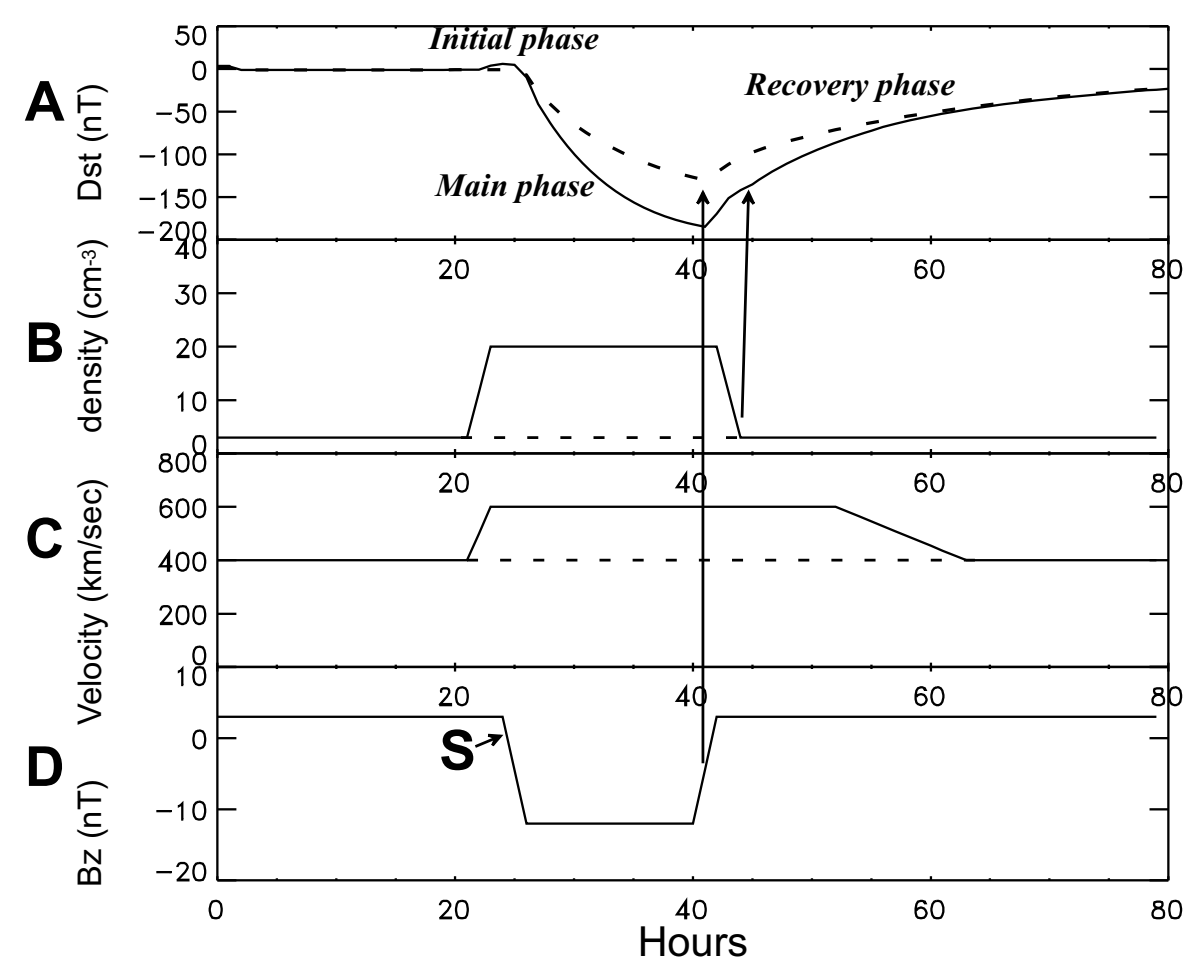

Fig. 5. Dst curves derived using model C with artificial input. (A) calculated Dst, (B) solar wind density, (C) solar wind velocity, and (D) IMF Bz. In each panel the dotted curve shows results calculated assuming no changes in velocity and density. The solid curve shows the results calculated using realistic changes.

sure and the electric field $(\mathbf{V} \times \mathbf{B z})$ induced by the IMF. As shown by the solid curve in Fig. 5(B), at 3 hours before $S$ the density increases from 3 to $20 \mathrm{~cm}^{-3}$ over 2 hours and stays there for 20 hours before falling back to $3 \mathrm{~cm}^{-3}$.

The solid line in panel $\mathrm{C}$ of Fig. 5 shows that 3 hours before $\mathrm{S}$ the velocity increased from 400 to $600 \mathrm{~km} \mathrm{~s}^{-1}$ in 2 hours, and stayed there for 30 hours, and then fell back to $400 \mathrm{~km} \mathrm{~s}^{-1}$ in 10 hours. The $\mathrm{Bz}$ time series is the same as for the previous simple case and is shown in Fig. 5(D). The calculated Dst is shown as a solid curve with an initial phase of about $+7 \mathrm{nT}$ in panel A. The minimum value is about $-185 \mathrm{nT}$ near the end of the period of negative Bz. The increased density and velocity resulted in a large Dst decrease during the period of negative $\mathrm{Bz}$.

\subsection{Density effect}

Figure 6 shows an extended summary of Fig. 5(A) for five cases of calculated model storms (dotted curves in Fig. 5), in which $\mathrm{Bz}$ remained at the $-12 \mathrm{nT}$ level for durations of 5, 10, ., 25 hours in 5-hour steps, and in which solar wind density and velocity are assumed to have no variations (Figs. 5(B) and 5(C)). Since there is no increase in the dynamic pressure in the input data, no initial phase increase is produced in the Dst calculated (as is evident in the dotted curve in Fig. 5(A)). At the end of the negative Bz period for each of the five cases, the calculated Dst time series has a minimum value corresponding to the final point of the main phase of the storm. For instance, for the case in which the duration of the Bz valley is 15 hours, the minimum Dst is about $-130 \mathrm{nT}$, as the dotted curve in Fig. 5(A) shows. The end of the negative Bz period signifies the beginning of the recovery phase as seen in Fig. 6 (and in Fig. 7 to be ex-

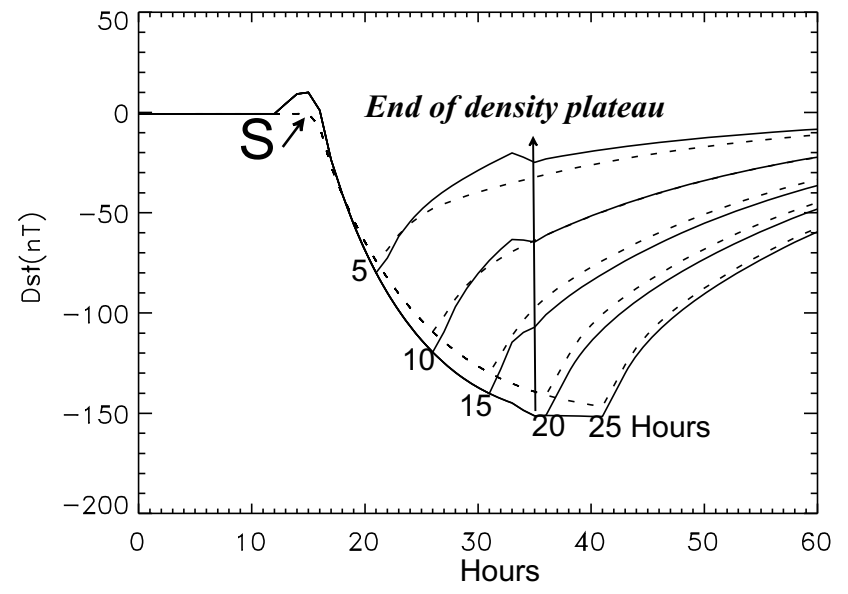

Fig. 6. Summary of storms that would occur during the period when $\mathrm{Bz}$ is $-12 \mathrm{nT}$, the period of minus $\mathrm{Bz}$ increases in 5-hour steps. The solid (dotted) curve shows Dst with a density plateau (without a plateau). One of the five cases having negative $\mathrm{Bz}$ valley of 15 hours in duration corresponds to the case of Fig. 5(A).

plained in the next section). The slope of the predicted Dst curve in the main phase becomes gentler as the duration of the $\mathrm{Bz}$ depression is lengthened. These curves resemble the exponential curves produced by a simple differential equation as has been shown by Burton et al. (1975). Usually, a northward turning of $\mathrm{Bz}$, which means the vanishing of the injection term $(\mathbf{V} \times \mathbf{B z}=0)$, stops further development of the storm. If the injection term continues to increase for a longer time, the decay term increases and becomes comparable with the injection term. Then Dst stops to develop and 


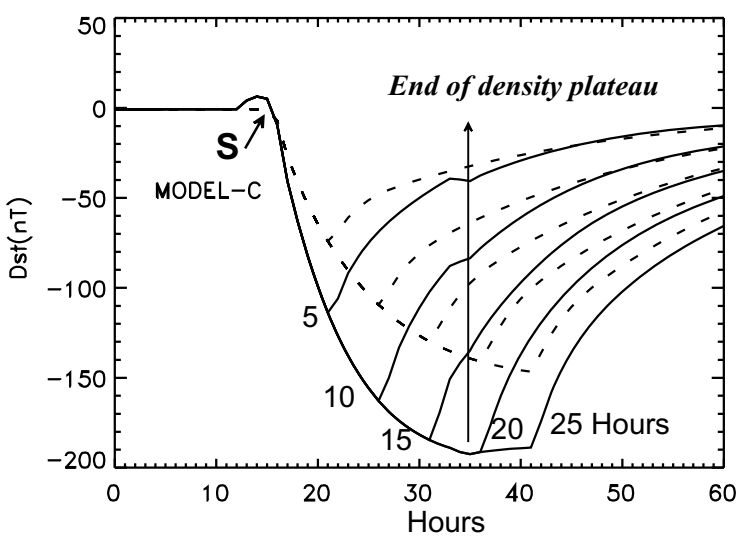

Fig. 7. Summary of Dst curves corresponding to Fig. 6 with a velocity plateau $(600 \mathrm{~km} / \mathrm{s})$ and a density plateau $\left(20 \mathrm{~cm}^{-3}\right)$.

begins to return towards more moderate levels.

Next, we input a time series that includes a density increase with a plateau at $20 \mathrm{~cm}^{-3}$ lasting for 20 hours as indicated by the solid curve in Fig. 5(B); however, the velocity is kept unchanged. The resultant five Dst time series are represented by solid curves in Fig. 6. A positive variation of about $10 \mathrm{nT}$ appears which corresponds to the initial phase. Take the case in which the negative $\mathrm{Bz}$ valley lasts for 15 hours. In this case, the density plateau makes the intensity of the storm, i.e., the minimum Dst, $10 \mathrm{nT}$ stronger than for the case of no density change.

These simple tests show that a solar wind density increase enhances the storm intensity. In the recovery phase (approximately during the period of positive $\mathrm{Bz}$ ) the density increase pushes the solid curve upward (toward less disturbance) until the end of the density increase; this feature is well demonstrated in Fig. 6 for the cases of 5, 10, and 15 hours of density increase. These properties of the calculated Dst curves correspond to well-known physical features of magnetic storms. In the initial phase, the higher dynamic pressure caused by an increase in the solar wind density makes the ChapmanFerraro current stronger and pushes the Dst curve up. A negative $\mathrm{Bz}$ causes a strong earthward $\mathbf{E} \times \mathbf{B}$ drift of the magnetospheric and plasma sheet plasma. The resulting enhanced convection provides the seed of the ring current by increasing the plasma sheet density with a certain time lag from the increase in the solar wind density (Ebihara and Ejiri, 2000). If the solar wind density effect in the main phase is stronger than the effect of the Chapman-Ferraro current, the Dst level would be lower than it would be if there were no density increase. The results shown in Fig. 6 seem to indicate that this is indeed the case. Figure 6 shows that each Dst curve has a different Dst level at the end of the density plateau relative to the level where the Dst is at when there is no density change (i.e., the dotted curves). In other words, the solid curves do not return exactly to the dotted curves at the end of density plateau. This indicates that Dst has a strong hysteresis.

\subsection{Velocity effect}

In Fig. 7 we show an extended summary representation of five pairs of model storms with and without velocity and density plateaus as indicated in Fig. 5. Usually, rises in velocity are accompanied by density increases as a solar

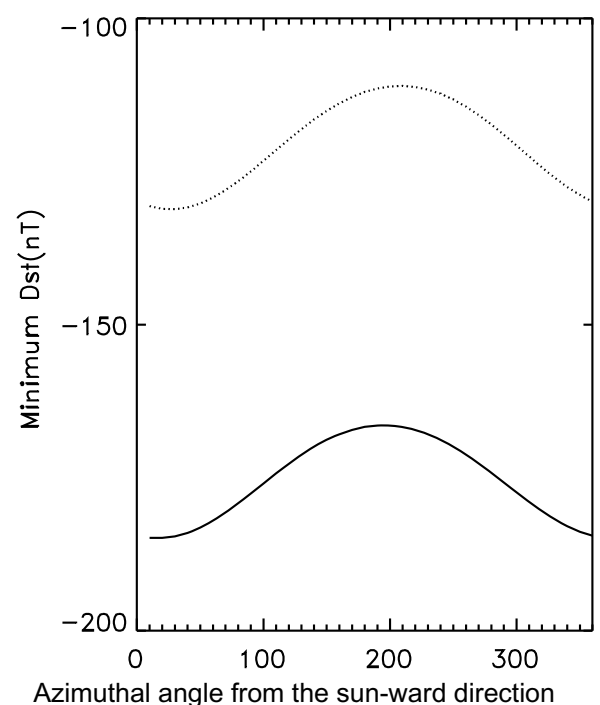

(degrees)

Fig. 8. Dependence of Dst on the azimuthal angle of the artificial interplanetary magnetic field.

wind with higher velocity compresses the preceding solar wind that has a lower velocity. Since a high velocity plateau without a density plateau that accompanies it seems to be unreal, and since the training time series included few such cases, we have not analyzed any cases of only a velocity plateau with no accompanying density plateau.

The dotted curves in Figs. 6 and 7 are the same and contain no density and velocity plateaus. The third solid curve in Fig. 7 represents the same case as the solid curve in Fig. 5(A). The length of the period in which $\mathrm{Bz}=-12 \mathrm{nT}$ is the same in Figs. 6 and 7. Clearly, a rise in velocity enhances the severity of a storm. In the third case, the storm having a negative Bz ( $-12 \mathrm{nT})$ for 15 hours had a Dst of about -185 nT (Fig. 7). Qualitatively, similar characteristics are found in the Dst behavior at the end of the density plateau in Figs. 6 and 7 . The effect of the velocity plateau on the dynamic pressure is similar to that of the density plateau, but in Fig. 7 the electric fields that increase the earthward plasma drift as part of the magnetospheric convection and that seed the ring current from the tail plasma sheet are more significant than the density effect in Fig. 6.

We then considered the effects of velocity during unusual periods. The minimum Dst during the 15-hour long negative $\mathrm{Bz}(-12 \mathrm{nT})$ periods that had only a velocity rise or only a density rise was about $-170 \mathrm{nT}$ or $-140 \mathrm{nT}$, respectively. Although the relative rise in velocity (from 400 to 600 $\mathrm{km} / \mathrm{sec}$ ) was much smaller than that of the density (from 3 to $20 \mathrm{~cm}^{-3}$ ), its effect was greater. In our physical analysis, we thus have to consider the hidden effect of the variable decay time for the ring current which depends on the velocity, density, and $\mathrm{Bz}$ in the $\mathrm{NN}$ analysis.

\subsection{Effect of the azimuthal angle of IMF}

By rotating the magnetic field components in the X-Y (GSM) plane for the artificial magnetic storm event shown in Fig. 5, we investigate the dependence of model $\mathrm{C}$ on the azimuthal direction of the magnetic field. Here the azimuthal angle is measured counterclockwise from the sunward direc- 


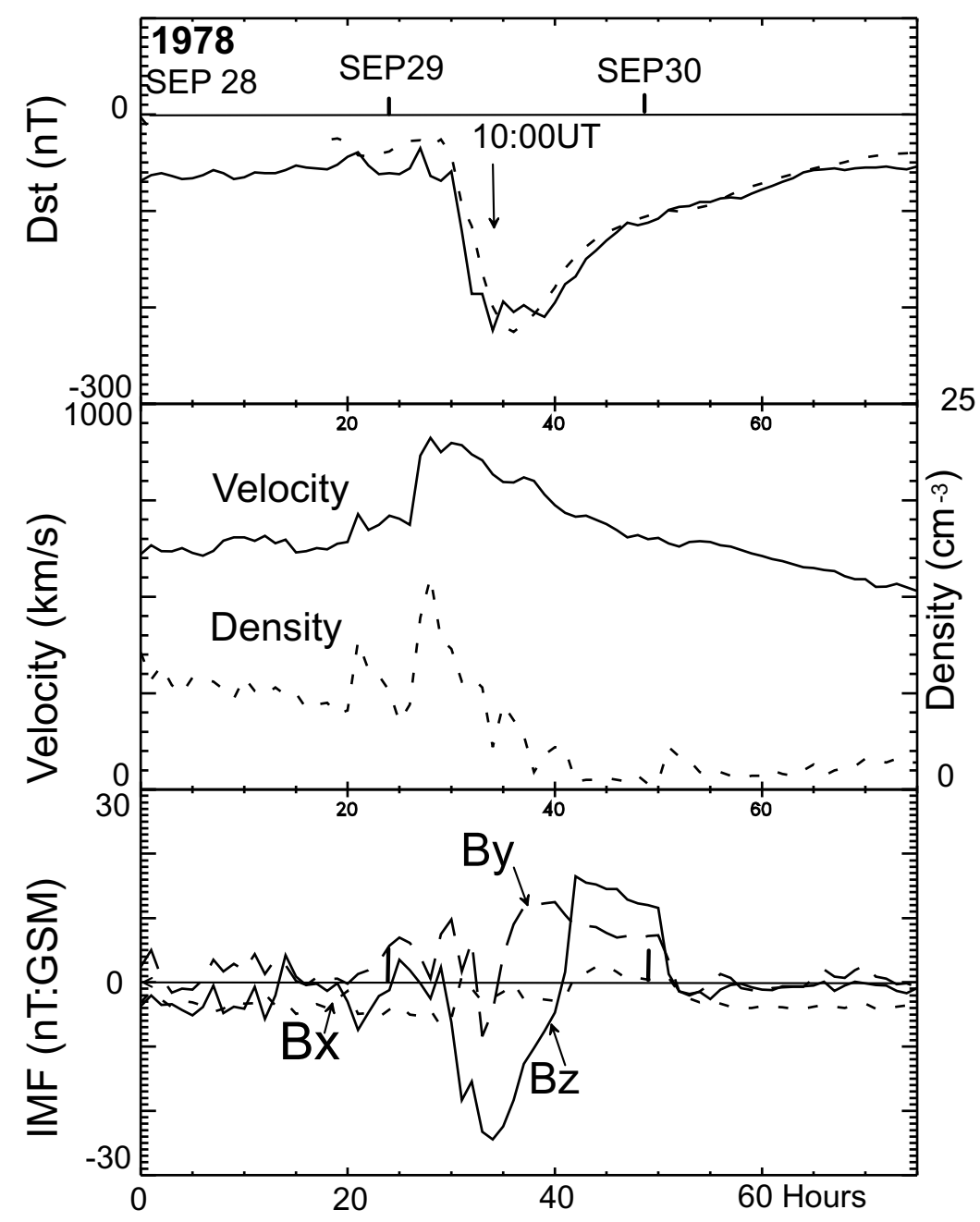

Fig. 9. Storm starting on 28 September 1978. The top panel: the actual Dst (solid curve) and the Dst predicted using model C (broken curve). The middle panel: solar wind velocity (solid curve) and density (dotted curve). The bottom panel: the Bx (dotted curve), By (broken curve), and Bz (solid curve) components of the IMF (GSM).

tion.

Figure 8 shows the dependence of the minimum Dst on the azimuthal angle of the magnetic field with a constant magnitude of $10 \mathrm{nT}$ in the X-Y plane. When the azimuthal angle of the magnetic field in the X-Y plane was in 10-30 degrees of the direction of the sun (positive X-direction), the magnitude of Dst appears to be greatest. The root-mean-square error for model $\mathrm{C}$ is about $10 \mathrm{nT}$ and the cross-correlation coefficient is over 0.9 when the solar wind data used in input are those taken near the earth. The difference between the minimum and maximum values of Dst calculated with varying azimuthal angle was about 20nT which is slightly above the quantitative prediction error. If we take $15 \mathrm{nT}$ as the magnetic field magnitude in the X-Y plane, the difference is over $30 \mathrm{nT}$. The larger the magnitude of the X-Y component the larger the difference in the calculated Dst.

Model B shows the same property, so we contend that this property qualitatively reflects the actual nature of storms. We use the actual velocity, density, and Bz values (but not those for Bx and By) from the strong storm on 29 September 1978 (a storm frequently referred to by researchers) as part of the training data (Fig. 9). When we calculated Dst for various azimuthal angles from the sunward direction un-

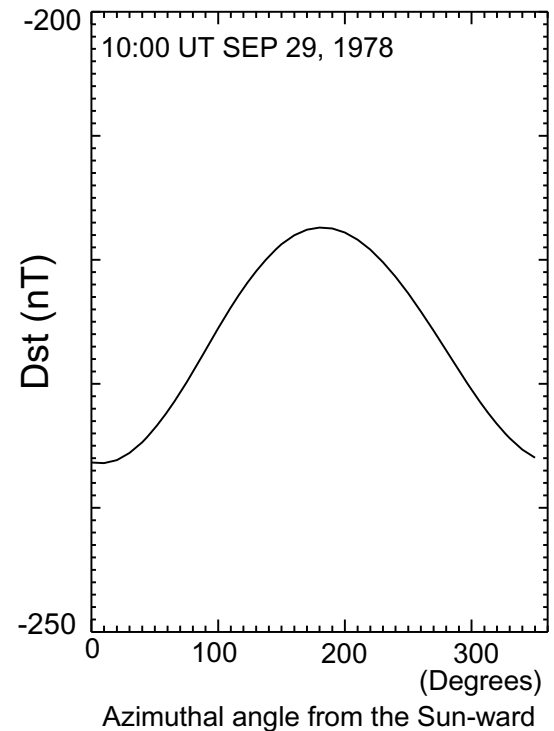

Fig. 10. Dependence of Dst on the azimuthal angle calculated from the solar wind parameters for the storm on 29 September 1978. 
der a fixed magnetic field component of $10 \mathrm{nT}$ in the X-Y plane (Fig. 10), the difference between the maximum and minimum of the peak Dst magnitude was about $20 \mathrm{nT}$. The minimum Dst (i.e., the maximum Dst magnitude) is obtained in the sunward direction.

Because Dst reaches a minimum at different times depending on the variable azimuthal angle in a complex storm, our analysis was made only for a simple storm. For 17 storms with a simple Dst variation which were selected from 1978 to 1982 , we calculated the azimuthal angle of IMF corresponding to the Dst minimum. Most of Dst minima occur regardless of the storm magnitude when IMF on the X-Y plane is within 45 degrees from zero azimuthal angle, i.e., the positive X direction. Models $\mathrm{B}$ and $\mathrm{C}$, and other models we have constructed, showed statistically similar properties for these storms.

\section{Discussion}

Many empirical differential equations have been formulated based on the injection function and decay time constant during the main and recovery phases of a storm. We examined the NN method using the classical model of Burton et al. (1975) and its revised version (O'Brien and McPherron, 2000) the use of which is restricted to $>-150 \mathrm{nT}$. The Burton model is frequently referred to in discussions on simulations of geomagnetic storms using differential equations. The model is as follows:

$$
\begin{aligned}
& \frac{d D s t^{*}}{d t}=F(E)-\frac{D s t^{*}}{\tau} \\
& D s t^{*}=D s t-b \sqrt{P}+c
\end{aligned}
$$

here

$$
\begin{aligned}
& F(E)=0 \quad E y<0.5 \mathrm{mV} / \mathrm{m} \\
& F(E)=d(E y-0.5) \quad E y>+0.5 \mathrm{mV} / \mathrm{m} \\
& b=16 \mathrm{nT}(\mathrm{nPa})^{-1 / 2}, \quad c=20 \mathrm{nT} \\
& d=-5.4 \times 10^{-3} \mathrm{nT}\left(\mathrm{mVm}^{-1}\right)^{-1} \mathrm{~s}^{-1} \\
& \tau=7.7 \text { hours } \\
& E y=V B s
\end{aligned}
$$

Dst* is the corrected Dst without the solar wind dynamic pressure $(P)$ and the ring current effect $(c)$ in a quiet period. $F(E)$ is the injection term, $V$ is the solar wind velocity, $B s$ is the southward component of the interplanetary magnetic field, and $\tau$ is the decay time. The parameter $\tau$ is important for obtaining a deeper insight into the physics of the processes that lead to particle losses from the ring current and for estimating the rate of energy input and the total energy input into the ring current during a magnetic storm.

We compared our NN model to the Burton model as a simple reference model. Because of the fine resolution used in the definition of Dst and for the sake of convenience for comparison of the models for space weather forecasting for a relatively short time span (1-8 hours) using data from the ACE spacecraft, we adopt one hour as the time resolution for the solar wind data. To solve Eq. (3) the Adams-BashforthMoulton method with a predictor and a corrector was used.

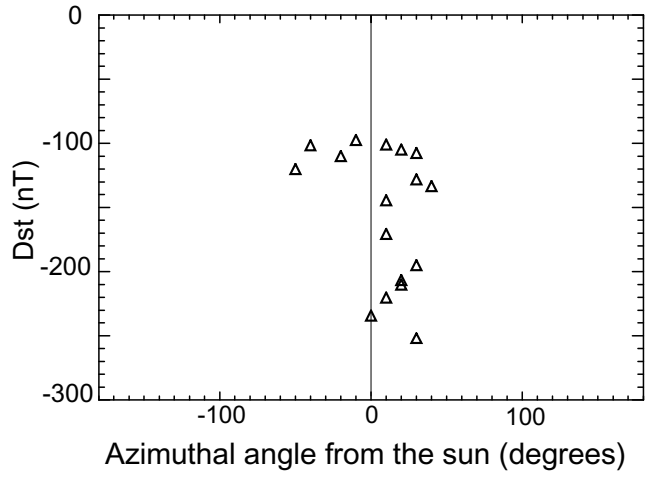

Fig. 11. Scatter plot of the minimum Dst as a function of the azimuthal angle measured from the sunward direction (in degrees) for storms with a simple Dst shape (1978 to 1982).

In many empirical models the value of $\tau$ depends on $\varepsilon$ input energy flux into the magnetosphere), $U$ (rate of energy supply to the ring current), and Dst (reviewed by Feldstein, 1992). We compare these models with the revised Burton model discussed by O'Brien and McPherron (2000) (which we refer to as the OM model) the applicability of which is limited to Dst $>-150 \mathrm{nT}$. The most important difference between the Burton model and the OM model is that $\tau$ depends on $E y(=V B s)$ in the OM model:

$$
\tau=2.40 \exp [9.74 /(4.69+V B s)] .
$$

Other coefficients are slightly changed so that:

$$
\begin{aligned}
& F(E)=0, \quad E y<E c=0.49 \mathrm{mV} / \mathrm{m}, \\
& F(E)=-4.4(V B s-E c), \quad E y>E c=0.49 \mathrm{mV} / \mathrm{m} \\
& D s t^{*}=D s t-7.26 P^{1 / 2}(\mathrm{nPa})+11 \mathrm{nT} .
\end{aligned}
$$

If $V B s$ is zero (Bz toward the north), $\tau$ reaches its maximum value (19.2 hours) in the OM model. To compare the NN, Burton, and OM models, we examined two storms that began on 10 October 1980 and on 2 March 1982, and that were analyzed by O'Brien and McPherron (2000) as their figures $8(\mathrm{a})$ and $8(\mathrm{~b})$. For these storms, the ground-station observations showed $-104 \mathrm{nT}$ and $-211 \mathrm{nT}$ for the minimum values of Dst. The decay time from the OM model is longer than the constant decay time ( 7.7 hours) obtained by the Burton model during the early main phase and in the recovery phase.

Panel A of Fig. 12 shows the Dst calculated by WDC Kyoto from the ground-station data beginning on 10 October 1980 (the thick gray curve), and the Dst predicted by the NN model (red curve), by the Burton model (green curve), and by the OM model (blue curve). The curve from the OM model corresponds to the multi-step curve given by O'Brien and McPherron (2000) for the same situation. To calculate the predictor in the Dst time series we need the four-step starting values. For the initial four-step values we used the actual data given by the ground stations. The starting time for the integration is denoted by an arrow which is 24 hours after the left edge of the panel. On the other hand, the calculation of the NN model started about 24 hours before the beginning for the Burton and OM models. The recurrent type NN model needs a longer time for the initial stage, especially 


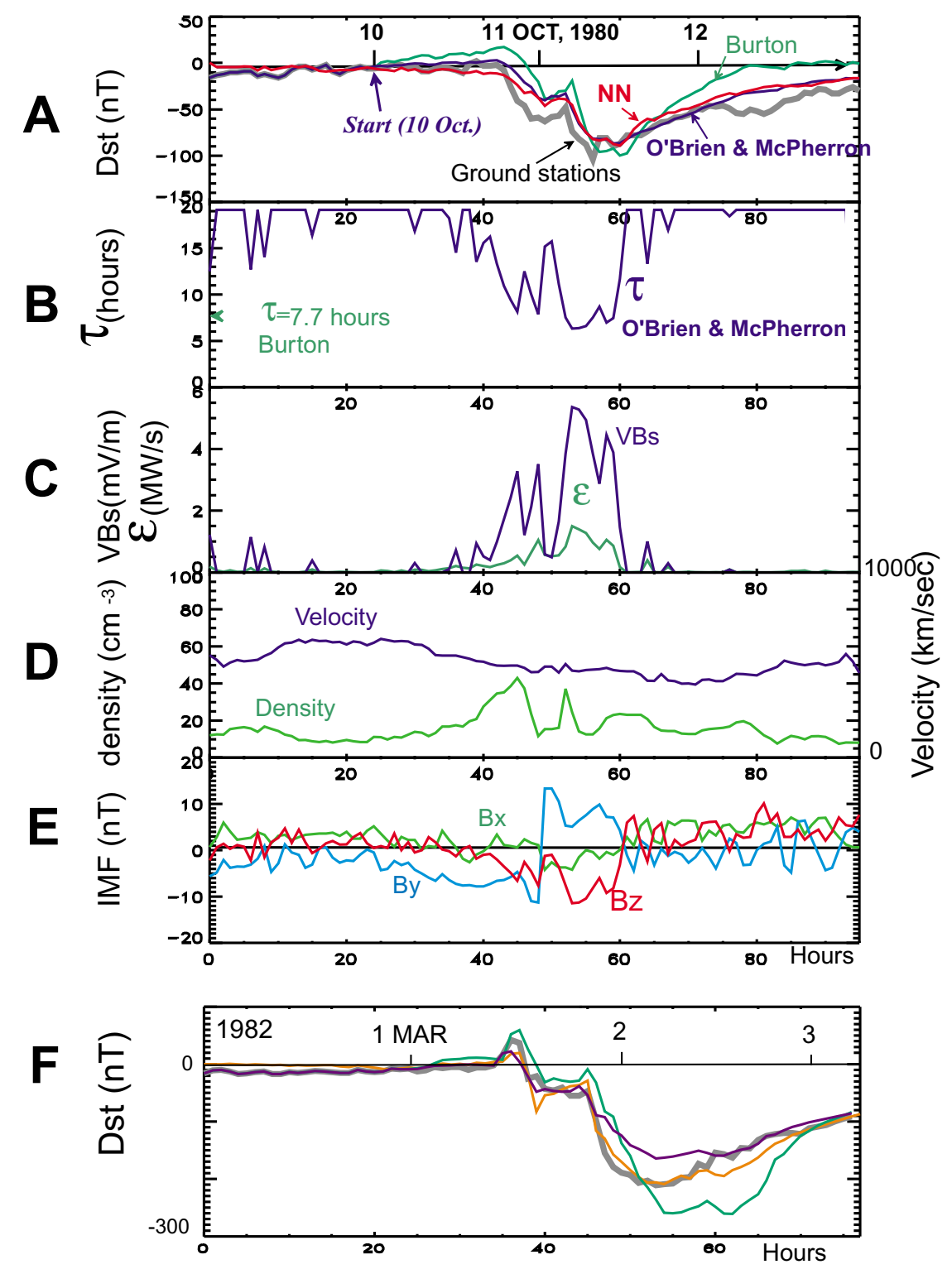

Fig. 12. Top panel noted A: Comparison of neural network model C (red), Burton (green), O'brian and MacPherron model (blue) and ground stations (fat gray) during 9 to 12 October 1980. Panel B: Decay time (hours) for the Burton (green), O'brian and MacPherron model (blue). Panel C: VBs and $\varepsilon$ of solar wind. Panel D: Velocity $(\mathrm{km} / \mathrm{s})$ and density $\left(\mathrm{cm}^{-3}\right)$ of solar wind. Panel E: Cartesian components (GSM) of the inter planetary magnetic field (nT). Panel F: The prediction curves by three models for the period of 28 February to 2 March 1982 including a large storm.

when the calculation starts with a large negative value of Dst especially when this happens to be in the recovery phase of a storm. However, since the actual operation of real-time prediction will continue for long periods of time (possibly several months or years), this problem is of little consequence.

The OM model predicts storms $(>-150 \mathrm{nT})$ as well as the NN model as is seen in panel A. Here we specifically report that the NN model works well for any event, which is an important factor for use in actual operational space weather forecasting. The calculated curves obtained from the Burton model appear to represent the Dst better when data with a fine time resolution (e.g., 2.5 minutes) are used than when data with a one-hour time resolution are used. The Burton model did not work well if data with a one-hour time resolution are used. We compared the three models with respect to a storm of 10 October 1980, which is the same storm as that shown in Fig. 8(a) of the article by O'Brien and McPherron
(2000). Essentially, the southward component of the IMF (Bz) shown in panel E determined the Dst index. As to the increasing magnitude of southward $\mathrm{Bz}$ we consider this to have happened in two steps and caused the disturbances of 10 October. Panel D shows the solar wind velocity $(\mathrm{km} / \mathrm{s})$ and density $\left(\mathrm{cm}^{-3}\right)$ with blue and green curves, respectively. In this event, no shock front of the solar wind was encountered by the satellite. During the period of gradual increase of the dynamic pressure of the solar wind, Bz was southward but its magnitude was small. In such cases the pressure increase in the initial phase is usually not obvious, as in the case discussed here. The first minimum of southward $\mathrm{Bz}$ that corresponds to the first Dst decrease of $-63 \mathrm{nT}$ which occurred at $01 \mathrm{UT}$ on 11 October. The second valley in Bz caused the minimum Dst of $-104 \mathrm{nT}$.

As is seen in panel A, the prediction curves from the NN and OM models fit the ground-based Dst curve fairly well. 
The Burton model does not fit the Dst during the initial and recovery phases of this storm. The OM model was given a longer decay time, $\tau$, than the Burton model $(\tau=7.7$ hours: constant) during the above mentioned period. Thus the Dst predicted by Burton model recovers more quickly than the prediction by the OM model as is expected from Eq. (3). The decay time that is set constant does not seem to represent the actual features of storms. This is a weak point of the Burton model. Earlier, Kamide and Fukushima (1971) had constructed models that used different values of $\tau$ in the main and recovery phases.

Using the energy injection rate, $\varepsilon$,

Akasofu (2001) has classified storms as follows:

Weak storms $\varepsilon \sim 0.25 \mathrm{MW} / \mathrm{s}$

Moderate storms $\varepsilon \sim 1.4 \mathrm{MW} / \mathrm{s}$

Very intense storms $\varepsilon \sim 8.0 \mathrm{MW} / \mathrm{s}$

Since $\varepsilon$ (green curve) in panel C reached almost $1.5 \mathrm{MW} / \mathrm{s}$, this storm was a 'moderate storm', according to Akasofu's scheme.

The storm of 1 March 1982 started at 11 UT with a clear initial phase caused by a strong shock wave. This storm is one of the cases studied by O'Brien and McPherron (2000). As shown in panel F of Fig. 12, the Burton model cannot predict the minimum Dst well. The OM model (blue curve) gives a better prediction. However, O'Brien and Mcpherron state that their model should not be used for storms having a Dst minimum less than $-150 \mathrm{nT}$. In the storm of March 1, 1982 the minimum was well bellow this value. Our NN does not have any model applicability limitation of this nature. The NN model represented by the red curve gives an even better prediction than the OM model.

We considered possible reasons for Dst varying with the direction of the interplanetary magnetic field in the GSM XY plane. The variation of Dst is mainly due to the changes of plasma injection (which is the seed of the ring current) from the plasma sheet to the night side inner magnetosphere. These changes are caused by the changes in the intensity of the convection electric field which is influenced by the $\mathrm{Z}$ and $\mathrm{Y}$ components of IMF. On the other hand, the X component of IMF might also control the amount of magnetosheath and plasma sheet plasma flow toward the earth. The reason for the prediction of Dst being quite accurate from the initial stage of the attempt in spite of many unsolved problems seems to be that Dst reflects the macroscopic structures of the solar wind.

An ideal model should be able to take into account all the prevailing conditions and should produce good predictions under any conditions. Our two-hour-prediction models for Dst, which use the Elman-type NN, were trained using six parameters: the velocity and density of the solar wind and the magnitude and the $\mathrm{Bx}, \mathrm{By}$, and $\mathrm{Bz}$ components of IMF. For our latest model, the time-series training data sets were constructed from 50 active periods and many quiet periods (44\% of the training time series), over 9058 hours in total, during the solar maximum-activity phase from 1978 to 1982 ; however, it has been found that the model is applicable to a much wider range of solar activity phases.

The NN method can in principle forecast Dst more than six hours ahead of time, but it takes an hour for the solar wind to travel from the location of L1 to the earth, and the time of strong response of the geo-magnetosphere is one hour. We therefore perform two-hour predictions. However, as longerterm predictions would also be valuable, we now have plans on constructing a longer-term prediction model using the NN method. Thus far we have not used enough parameters in calculating Dst; in the future we should include as inputs the fluctuation terms, the temperature, and solar activity. Also, use of other neural-network methods, especially nonrecurrent $\mathrm{NNs}$, should be tested in a future program.

\section{Summary}

In this section we summarize our results.

(i) Using real-time data from the ACE spacecraft, we have constructed a prediction model for Dst, which has been working well since May 1998 (http://www.crl.go.jp/uk/uk223/ service/nnw/index.html).

(ii) During the period from February to October 1998, there were eleven storms which had a minimum Dst of less than $-80 \mathrm{nT}$. For ten of these storms the differences between the minimums Dst predicted using ACE data and the minimum Dst calculated from data collected by ground stations was less than about $23 \%$. For the remaining storm, which started on 19 October 1998, the difference was $48 \%$, perhaps because the solar wind factors prevailing near the ACE spacecraft and those in the vicinity of the earth were correlated only moderately well.

(iii) The principal source of geomagnetic disturbances has again been confirmed to be the $\mathrm{Bz}$ component of IMF. However, a positive $\mathrm{Bx}$ component seems to increase the magnitude of Dst. The NN prediction model might be useful as a simple simulator to examine minor effects such as those of Bx and By. Dst seems to be enhanced when the direction of IMF is toward the sun.

(iv) An increased solar wind density tends to produce a positive change in the Dst index through the ChapmanFerraro current in the initial and recovery phases (Fig. 6). On the other hand, the solar wind density enhances Dst almost $10 \%$ from the case of no density increase plateau in the main phase when $\mathrm{Bz}$ is negative.

(v) The velocity effect appears to be stronger than that of the density according to our NN model.

(vi) Our operational model, in principle, has no limitation in application perhaps excepting storms of extreme intensity.

Acknowledgments. We thank the ACE SWEPAM instrument team, the ACE MAG instrument team and the ACE Science Center for providing ACE data. We acknowledge that the criticisms from the reviewers were helpful in improving the paper. We thank M, Sugiura for his critical reading of the manuscript.

\section{References}

Akasofu, S.-I., Predicting Geomagnetic Storms as a Space Weather Project, Geophys. Monogr. Ser., 125, edited by P. Song, H. J. Singer, and G. L. Siscoe, AGU Washington DC, 329-337, 2001. 
Blanchard, G. T. and R. L. McPherron, A bimodal representation of the response function relating the solar wind electric field to the $\mathrm{AL}$ index, Solar-Terrestrial Prediction Proc., vol. 2, pp. 479-486, U. S. Dept. of Commerce, Boulder, Colo., 1992.

Burton, R. K., R. L. McPherron, and C. T. Russel, An empirical relationship between interplanetary conditions and Dst, J. Geophys. Res., 80, 42044214, 1975.

Ebihara, Y. and M. Ejiri, Modeling of solar wind control of the ring current buildup: A case study of the magnetic storms in April 1997, Geophys. Res. Lett., 25, 3751-3754, 1998.

Ebihara, Y. and M. Ejiri, Simulation study on fundamental properties of the storm-time ring current, J. Geophys. Res., 105, 15,843-15,859, 2000.

Elman, J. L., Finding structure in time, Cognitive Sci., 14, 179, 1990.

Feldstein, Y. I., Modeling of the magnetic field of magnetospheric ring current as a function of interplanetary medium parameters, Space Sci. Rev., 59, 83-165, 1992.

Iyemori, T., H. Maeda, and T. Kamei, Impulse response of geomagnetic indices to interplanetary magnetic field, J. Geomag. Geoelectr., 31, 1-9, 1979.

Kamide, Y. and N. Fukushima, Analysis of magnetic storms with DRindices for equatorial ring current field, Rep. Ionoshere Space Res. Japan, 25, 125-162. 1971.

Klimas, A. J., D. Vassiliadis, and D. N. Baker, Data-derived analogues of the magnetospheric dynamics, J. Geophys. Res., 102, 26,993-27,009, 1997.

Klimas, A. J., D. Vassiliadis, and D. N. Baker, Dst index prediction using data-derived analogues of the magnetospheric dynamics, J. Geophys. Res., 103, 20,435-20,447, 1998.

Kugblenu, S., S. Taguchi, and T. Okuzawa, Prediction of the geomagnetic storm associated Dst index using an artificial NN algorithm, Earth Planet Sci., 51, 307-313, 1999.

Lundstedt, H. and P. Wintoft, Prediction of geomagnetic storms from solar wind data with the use of a neural network, Ann. Geophys., 12, 19-24, 1994.

McComas, D. J., S. J. Bame, P. Barker, W. C. Feldman, J. L. Phillips, P. Riley, and J. W. Griffee, Solar wind electron proton alpha (SWEPAM) for the advanced composition explorer, Space Sci. Rev., 86, 563-612, 1998.

O'Brien, T. P. and R. L. McPherron, An empirical phase space analysis of ring current dynamics: Solar wind control of injection and decay, $J$. Geophys. Res., 105, 7707-7719, 2000.

Smith, C. W., J. L'Heureux, N. F. Ness, M. H. Acuna, L. F. Burlaga, and J. Scheifele, The ACE magnetic fields experiment, Space Sci. Rev., 86, 613-631, 1998.

Wu, J.-G. and H. Lundstedt, Geomagnetic storm predictions from solar wind data with the use of dynamic neural networks, J. Geophys. Res., 102, 14,255-14,268, 1997a.

Wu, J.-G. and H. Lundstedt, Neural network modeling of solar windmagnetosphere interaction, J. Geophys. Res., 102, 14,457-14,466, $1997 \mathrm{~b}$. Zwickl, R. D., K. A. Dogget, S. Sahm, W. P. Barrett, R. N. Grubb, T. R. Detman, V. J. Raben, C. W. Smith, P. Riley, R. E. Gold, R. A. Mewaldt, and T. Maruyama, The NOAA real-time solar-wind (RTSW) system using ACE data, Space Sci. Rev., 86, 633-648, 1998.

S. Watanabe (e-mail: watanabe@crl.go.jp), E. Sagawa, K. Ohtaka, and H. Shimazu 